\title{
Frequency Offset Estimation Based on PRACH Preambles in LTE
}

\author{
Aijun Cao, Pei Xiao, Rahim Tafazolli \\ \{a.cao,p.xiao,r.tafazolli\}@surrey.ac.uk \\ Centre for Communication Systems Research \\ Department of Electronic Engineering, University of Surrey \\ Guildford, Surrey, GU2 7XH, United Kingdom
}

\begin{abstract}
This paper presents a novel method to estimate the frequency offset between a mobile phone and the infrastructure when the mobile phone initially attaches to the LTE network. The proposed scheme is based on PRACH (Physical Random Access Channel) preambles and can significantly reduce the complexity of preamble detection at the eNodeB side.
\end{abstract}

\section{INTRODUCTION}

With the increased demand for higher data rate provided by mobile communication systems, multi-carrier techniques are adopted to the next generation industry standard, such as OFDM for downlink and SC-FDMA for uplink in the Long Term Evolution (LTE) system. LTE is an industrial standard, aiming to provide high data rate wireless services and cope with future technology evolutions. One of the goals of LTE is improving spectral efficiency. The orthogonal subcarriers provide more degrees of freedom to allocate the radio resource compared to the previous cellular standards, leading to improved spectral efficiency. But one inherent disadvantage of OFDM is its sensitivity to frequency errors, which destroys the orthogonality among sub-carriers, causing performance degradation.

As an initial step to connect to the network, one UE sends out a preamble chosen by the standardized procedure, and the eNodeB which the UE tries to attach needs to detect the preamble and obtain an initial estimate of the frequency offset between the UE and the eNodeB (coarse frequency synchronization). Different frequency offset estimation methods were proposed in the literature, e.g., blindly [3] - [7] or based on training symbols [8] - [12]. Note that there is only one occurrence of a random access preamble transmission in each access attempt, thus it is not enough for those methods to obtain a good performance in the initial step. A practical solution is to partition the possible range of frequency offset into several frequency bins, and perform the preamble detection in each frequency bin. The possible range is determined based on the requirements for the oscillator accuracy specified in 3GPP standards and the maximum supported velocity of the eNodeB, as well as the supported carrier frequency etc. For example, suppose that UEs oscillator accuracy is $\pm 0.1 \mathrm{ppm}$ and eNodeBs oscillator accuracy is $\pm 0.05 \mathrm{ppm}$ [1], and the maximum supported velocity is $200 \mathrm{~km} / \mathrm{h}$, and the carrier frequency is $2 \mathrm{GHz}$. In this case, the range is $[-670,670] \mathrm{Hz}$. If the bin width is $50 \mathrm{~Hz}$, then there are 28 bins in total, and eNodeB performs preamble detections in each bin for the same access interval.

This straightforward method does not require a separate frequency offset estimator for each bin. However, it requires number of bins times of preamble detection trials, thus the complexity is proportional to the number of bins.

In this paper, we propose a novel scheme for coarse frequency synchronization by utilizing some special property of the random access preambles in LTE. The remainder of the paper is organized as follows. Sec. II describes the system model. The proposed frequency offset estimation algorithm is presented in Sec. III. The simulation results are shown in Sec. IV. Finally, conclusions are drawn in Sec. V.

\section{SYSTEM MODEL}

\section{A. Random Access Preamble}

A preamble signal is defined according to [2] as

$$
s(t)=\beta_{\mathrm{PRACH}} \sum_{k=0}^{N_{\mathrm{ZC}}-1} \sum_{n=0}^{N_{\mathrm{ZC}}-1} x_{u, v}(n) \cdot e^{-j \frac{2 \pi n k}{N_{\mathrm{ZC}}}} \cdot e^{j 2 \pi\left(k+\varphi+K\left(k_{0}+1 / 2\right)\right) \Delta f_{\mathrm{RA}}\left(t-T_{\mathrm{CP}}\right)}
$$

where

- $\quad \beta_{\text {PRACH }}:$ determines the transmit power

- $\quad N_{\text {ZC }}$ : length of root sequence, 839 for FDD LTE

- $\Delta f_{\mathrm{RA}}$ : bandwidth of one subcarrier for preamble signals, $1.25 \mathrm{kHz}$

- $\quad K: 15 \mathrm{kHz} / \Delta f_{\mathrm{RA}}=15 / 1.25=12$

- $k_{0}$ : determines over which band the preamble is transmitted

- $\quad \psi$ : a predefined constant

- $\quad x_{u, v}(n)$ : preamble sequence which is derived from $x_{u, v}(n)=x_{u}\left(\left(n+C_{v}\right) \bmod N_{\mathrm{ZC}}\right)$

where $x_{u}(n)=e^{-j \frac{\operatorname{mun}(n+1)}{N_{\mathrm{ZC}}}}, \quad 0 \leq n \leq N_{\mathrm{ZC}}-1$, and $u$ is the root sequence number, and $C_{v}$ is a value from a predefined set.

- $\quad T_{\mathrm{CP}}$ : duration of the cyclic prefix (CP) 
To facilitate analysis and without loss of generality, we assume:

$$
\begin{aligned}
- & k_{0}=0 \\
- & \Psi=0 \\
- & C_{v}=0
\end{aligned}
$$

The $1 / 2$ sub-carrier fixed frequency offset can be ignored since it is known and can be compensated at the receiver side. And furthermore, we assume that the channel gains of $\mathrm{N}_{\mathrm{zc}}$ sub-carriers are approximately similar. If we only look at those discrete points with the sampling rate $\left(N_{Z C} \cdot \Delta f_{\mathrm{RA}}\right)$ after removal of the cyclic prefix, i.e., $t=\frac{n}{\left(N_{Z C} \cdot \Delta f_{\mathrm{RA}}\right)}+T_{C P}$

The normalized carrier frequency offset over the sampling rate between the UE and the eNodeB is $\Delta f$. Define $\Delta \omega=2 \pi \Delta f$ then the $n^{\text {th }}$ received preamble signal samples $s(n)$ can be simplified and approximated as

$$
s(n)=H \beta_{\mathrm{PRACH}} e^{j \Delta \omega \cdot n} e^{-j \frac{\pi u n(n+1)}{N_{\mathrm{ZC}}}}+q(n),{ }_{(2)}
$$

where $H$ is the channel gain, and $q(n)$ is the noise.

\section{B. Preamble Detector}

Fig. 1 shows the proposed preamble detector for one root sequence. The main task for the preamble detector is not only to detect all the cyclic shifts of the transmitted preambles by possibly multiple UEs at the same access interval for the concerned root sequence number, but also to estimate the initial carrier frequency offset between the UE and the eNodeB.

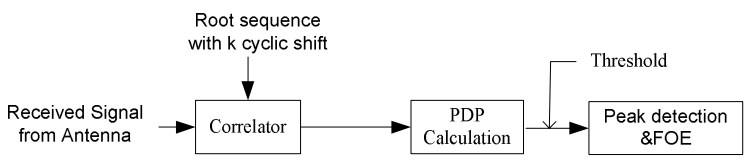

Figure 1 A preamble detector for one root sequence

The output of the preamble correlator is the inner product of the received vector and the conjugate of the associated Zadoff-Chu sequence with a certain cyclic shift $k$, i.e.,

$$
\begin{aligned}
& C(k)=\sum_{n=0}^{N_{z c}-1} s(n) e^{j \frac{\pi u(n+k)(n+k+1)}{N_{z c}}} \\
& =\sum_{n=0}^{N_{z c}-1}\left(H \beta_{\mathrm{PRACH}} e^{j \Delta \omega n n} e^{-j \frac{\pi u n(n+1)}{N_{\mathrm{zc}}}}+q(n)\right) e^{j \frac{\pi u(n+k)(n+k+1)}{N_{z c}}} \\
& =H \beta_{\mathrm{PRACH}} \frac{e^{j \frac{\pi u}{N_{z c}}\left(k^{2}-k\right)}\left(1-e^{j N_{z c} \Delta \omega}\right)}{1-e^{j\left(\Delta \omega-\frac{\pi u}{N_{z c}} k\right)}+q^{\prime}(n)}
\end{aligned}
$$

Let

$$
C v(k)=\frac{e^{j \frac{\pi u}{N_{z c}}\left(k^{2}-k\right)}\left(1-e^{j N_{z c} \Delta \omega}\right)}{1-e^{j\left(\Delta \omega-\frac{\pi u}{N_{z c}} k\right)}},
$$

the power of $C v(k)$ can be obtained as

$$
P(k)=\left|C_{v}(k)\right|^{2}=\frac{1-\cos \left(N_{z c} \Delta \omega\right)}{1-\cos \left(\frac{\pi u}{N_{z c}} k-\Delta \omega\right)}
$$

Fig. 2 illustratesan example of $P(k)$ when $\mathrm{f}_{0}=500 \mathrm{~Hz}$.

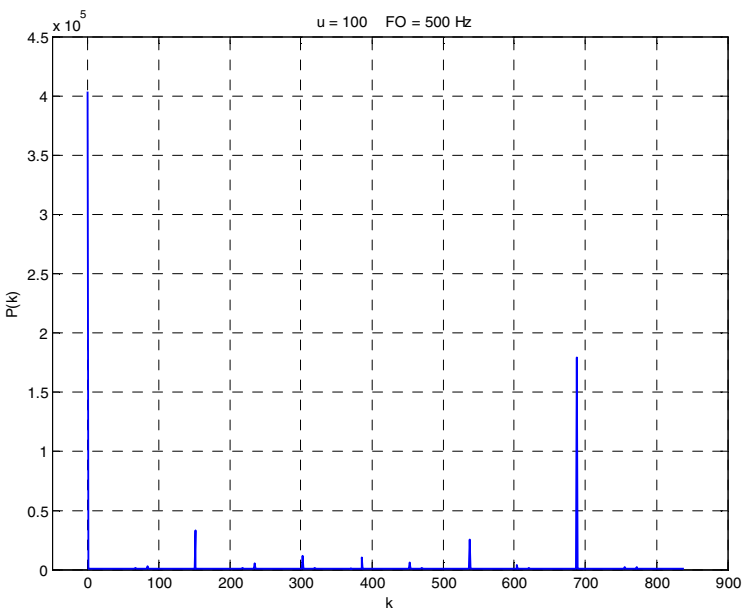

Figure $2 \mathrm{P}(\mathrm{k})$ peaks due to the frequency offset

It can be observed Fig. 2 that there are a certain number of peaks in $P(k)$ due to the frequency offset, and the positions and the values of peaks are determined by the root sequence number $u$ and the actual frequency offset.

\section{FREQUENCY OFFSET ESTIMATOR BASED ON PREAMBLES}

The main idea of the proposed scheme is to perform frequency offset estimation after preamble detection based on Eq.(5). Two methods will be described in the sequel. Note that we focus on the fractional frequency offset estimation. For the integer times of the sub-carrier of the frequency offset, it can be solved easily by circulate shifting.

\section{A. Peak ratio based estimate (PRE)}

One way to estimate frequency offset is to use the ratio of some peak values, e.g., the largest peak value over the second largest peak value, since the largest peak and the second largest peak are most resistant to noise. The ratio contains information about the frequency offset, thus the frequency offset can be estimated from the ratio. This is called Peak Ratio based Estimation (PRE).

Obviously the following can be observed from Eq. (5) about the positions of the peaks:

- All peaks show up at the even positions ( $k$ is even).

- The position of the largest peak, labelled as $\beta_{1}$, is $\beta_{1}=0$.

- The position of the second largest peak, labelled as $\beta_{2}$, should be $2 * m$, where $\bmod \left(m^{*} u, N z c\right)=1 ; \mathrm{e} . \mathrm{g}$, for $u=100, N z c=839, m$ should be 344 , thus the second position $\beta_{2}$ is at $688=2 * 344$. 
In order to perform PRE, we need to define a function $R$ which is the ratio of the largest and the second largest peaks as a function of the actual frequency offset. This can be generated according to Eq. (5) and $\beta_{1}, \beta_{2}$ for each root sequence number:

$$
R(\Delta f)=\frac{1-\cos \left(\frac{\pi u \beta_{2}}{N_{z c}}-2 \pi \Delta f\right)}{1-\cos (2 \pi \Delta f)}
$$

From the above equation, the normalized carrier frequency offset can be estimated as

$$
\begin{aligned}
& \Delta f= \\
& \frac{1}{2 \pi}\left[\cos ^{-1}\left(\frac{R-1}{\sqrt{R^{2}-2 R \cos \left(\frac{\pi u \beta_{2}}{N_{Z c}}\right)+1}}\right)-\cos ^{-1}\left(\frac{R-\cos \left(\frac{\pi u \beta_{2}}{N_{z c}}\right)}{\sqrt{R^{2}-2 R \cos \left(\frac{\pi u \beta_{2}}{N_{Z c}}\right)+1}}\right)\right](7)
\end{aligned}
$$

The following figure shows one example for $u=100$ :

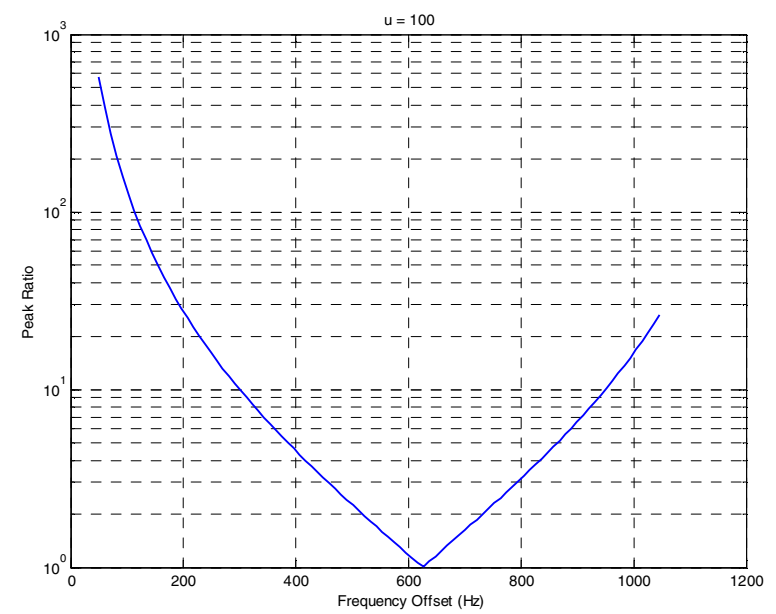

Figure 3 Ratio Vs frequency offset

Fig. 3 indicates that with the increase of frequency offset values of the largest peak and the second largest peak become closer and closer, until at the half of the sub-carrier spacing $625 \mathrm{~Hz}$ they become equal, and when the frequency offset continues increasing, the peak value at $\beta_{2}$ becomes higher than that at $\beta_{1}$.

As indicated by Fig. 3, the detected largest peak could be either the real largest peak $\beta_{1}$ with a frequency offset of less than $625 \mathrm{~Hz}$, or $\beta_{2}$ with a frequency offset of larger than $625 \mathrm{~Hz}$. This ambiguity could not be resolved by the PRE scheme itself. However, since the frequency offset usually is less than half subcarrier spacing, the PRE method would work in most practical situations.

\section{B. Peak difference based estimate (PDE)}

Similarly to the PRE method, the difference between the largest peak and the second largest peak rather than the ratio can be applied. This is called Peak Difference based Estimation (PDE), an example of which is shown in Fig. 4.
Because the difference varies for different signal-to-noise ratio (SNR) of the preambles, the difference can be normalized to the received signal power as:

$$
\lambda=\frac{P_{1}-P_{2}}{\rho}=D(\Delta f)
$$

where

- $\quad \lambda$ : normalized peak difference calculated.

- $\quad P_{1}, P_{2}:$ the largest and second largest peak values

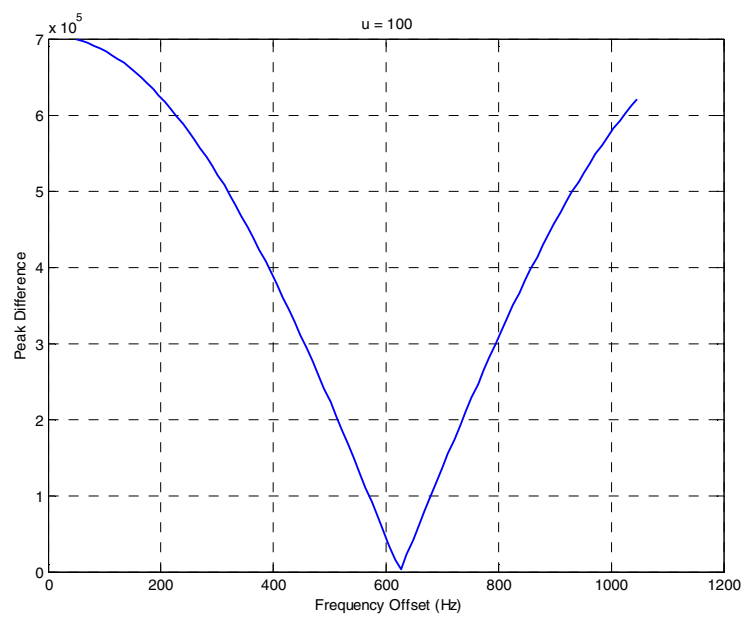

Figure 4 Difference Vs frequency offset

- $\quad \rho$ : received signal power of the preambles, it can also be obtained during preamble detection

- $D(\Delta f)$ : function between the difference and the frequency offset according to Eq. (5).

$$
D(\Delta f)=\frac{1-\cos \left(2 \pi N_{z c} \Delta f\right)}{1-\cos (2 \pi \Delta f)}-\frac{1-\cos \left(2 \pi N_{z c} \Delta f\right)}{1-\cos \left(\frac{\pi u}{N_{z c}} \beta_{2}-2 \pi \Delta f\right)}
$$

In order to facilitate the implementation, $D(\Delta f)$ can be approximated by a second-order polynomial:

$$
D(\Delta f)=A \cdot(\Delta f)^{2}+B \cdot(\Delta f)+C,
$$

where $A, B$ and $C$ are calculated from Eq. (5) for each root sequence using curve fitting, e.g., for $u=100, A=-1.5185, B$ $=-228.4243, C=7.2419 \mathrm{e} 5$, as illustrated in Fig. 5 . 


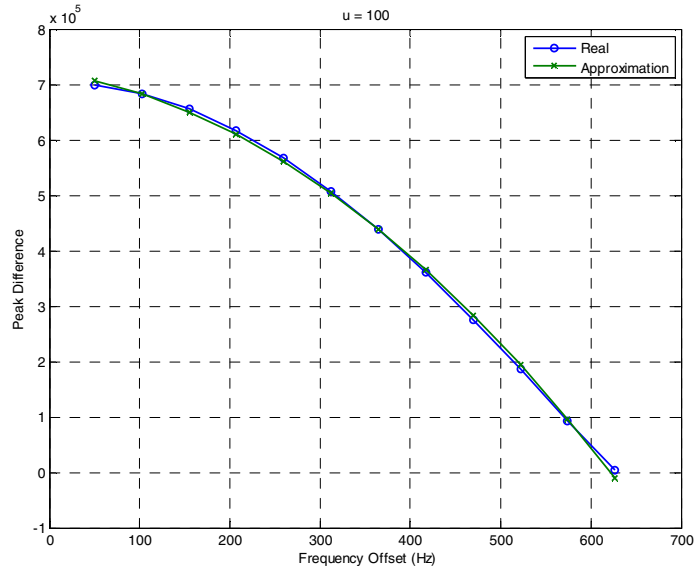

Figure 5 Difference Vs frequency offset

Based on Eqs. (6) and (7), the frequency offset estimate can be derived as

$$
\Delta f=\frac{B+\sqrt{B^{2}-4 A(C-\lambda)}}{-2 A}
$$

Similar to PRE, PDE can also only work up to $625 \mathrm{~Hz}$.

\section{NUMERICAL RESULTS}

Under the simulation setup shown in table 1, the performances of both PRE and PDE, as well as the frequency bin method, are simulated under ETU (Extended Typical Urban model) [1] multipath fading channel with $70 \mathrm{~Hz}$ Doppler frequency, as shown in Fig. 6 for different SNRs, where Figs. 6(a), 6(b) and 6(c) show the mean and normalized variance of the frequency offset estimate varies along with the real frequency offset under $\mathrm{SNR}=1 \mathrm{~dB}, 5 \mathrm{~dB}$, and $10 \mathrm{~dB}$ respectively. In each sub-figure, there are three curves corresponding to the performance of PRE, PDE and the frequency bin method with a bin size of $50 \mathrm{~Hz}$.

Table-1 Simulation Setup

\begin{tabular}{|c|c|c|c|}
\hline Parameter & Value & Parameter & Value \\
\hline $\mathrm{N}_{\mathrm{zc}}$ & 839 & $\mathrm{u}$ & 100 \\
\hline$\Delta \mathrm{f}$ & $1250 \mathrm{~Hz}$ & Channel & ETU \\
\hline $\mathrm{FFT}$ & 1024 & Doppler & $70 \mathrm{~Hz}$ \\
\hline $\mathrm{CP}$ & 128 & & \\
\hline
\end{tabular}

It could be observed from Fig. 6 that the performance of PDE is slightly worse than that of PRE. This follows from the fact that PDE requires estimation of the received preamble power. Furthermore, one can see from the figure that PRE is preferable to PDE by achieving a comparable performance to the conventional frequency bin method, but with much less complexity. Table- 2 illustrates the comparison of the required computations for one preamble detection between PRE and frequency bin method to achieve the performances shown in Figs. (6).

Table-2 Required computation comparison

\begin{tabular}{|c|c|c|c|c|c|c|}
\hline & $\begin{array}{c}1024- \\
\text { IFFT }\end{array}$ & $\begin{array}{c}\text { Complex } \\
\text { Multi. }\end{array}$ & $\begin{array}{c}\text { Complex } \\
\text { Addition }\end{array}$ & $\begin{array}{c}\text { Real } \\
\text { Multi. }\end{array}$ & $\begin{array}{c}\text { Real } \\
\text { Addition }\end{array}$ & $\begin{array}{c}\text { Real } \\
\text { Division }\end{array}$ \\
\hline $\begin{array}{c}\text { Freq. } \\
\text { Bin }\end{array}$ & 28 & 28672 & 28644 & - & - & - \\
\hline PRE & 1 & 1024 & 1023 & 87 & 23 & 19 \\
\hline
\end{tabular}

Therefore PRE strikes a good balance between implementation cost and achievable performance.
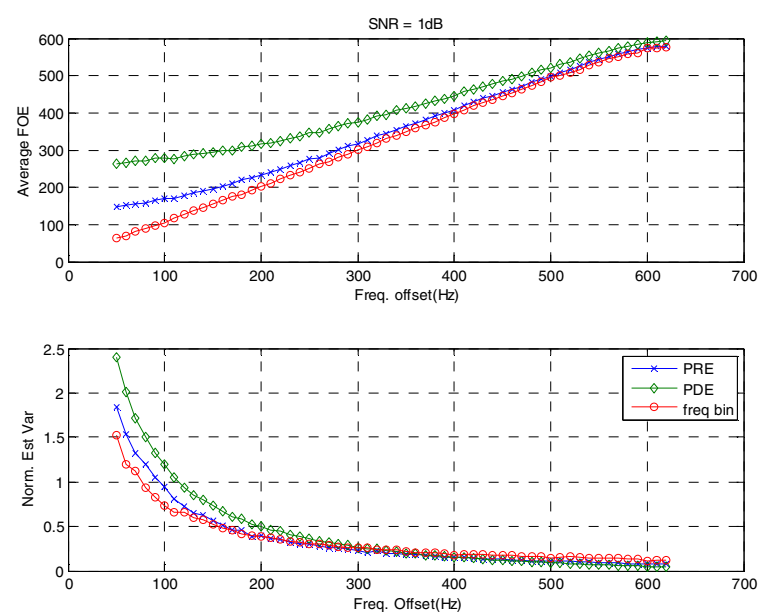

(a)
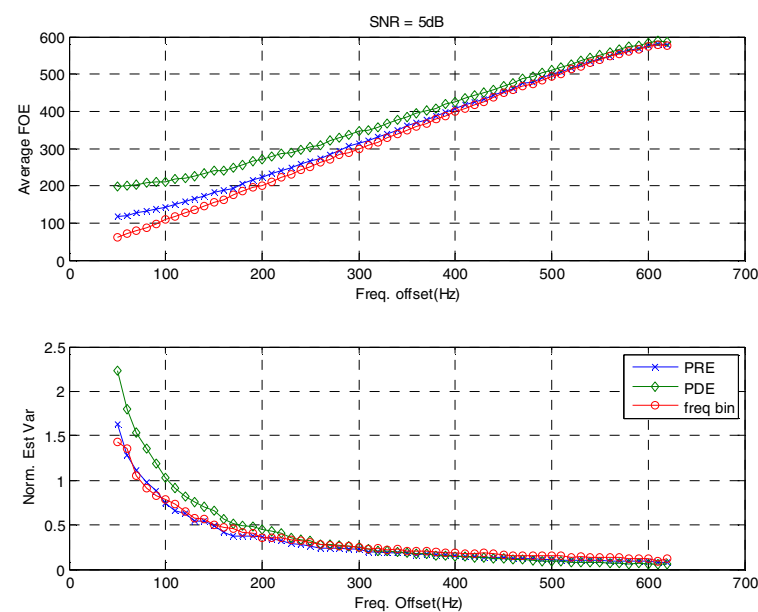

(b)
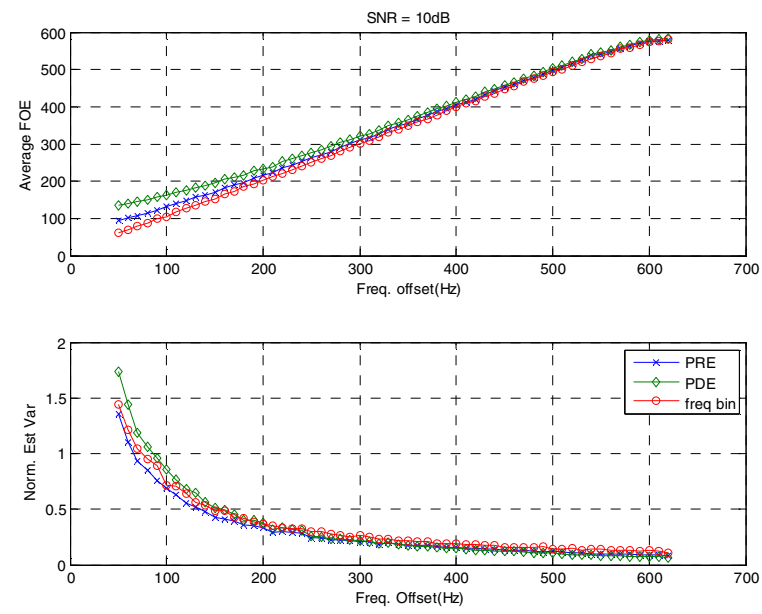

(c)

Figure 6 PRE, PDE performance under ETU fading channel 


\section{CONCLUSIONS}

This paper proposes a method to perform an initial frequency offset estimation by utilizing the preamble detection outputs with some simple additional operations. As such, the initial frequency offset estimates can be obtained at the same time when the preamble is detected. It can achieve coarse frequency synchronization while significantly reducing the implementation cost.

\section{ACKNOWLEDGEMENT}

The work presented in this paper is based on an international patent "Apparatus and methods for estimating and correcting frequency offset in LTE" (with reference number WO2010040264) which has been filed by ZTE.

\section{REFERENCES}

[1] 3GPP Technical Specification 36.104, '(Release 10)', www.3gpp.org.

[2] 3GPP Technical Specification 36.211, 'Physical channels and modulation(Release 10)', www.3gpp.org.

[3] T. M. Schmidl and D. C. Cox, "Blind synchronization for OFDM", Electron. Lett., vol. 33, no. 2, pp. 113-114, Jan. 1997.

[4] H. Liu and U. Tureli, "A high-efficiency carrier estimator for OFDM communications", IEEE Commun. Lett., vol. 2, no. 4, pp. 104-106, Apr. 1998.

[5] U. Tureli, H. Liu, and M. D. Zoltowski, "OFDM blind carrier offset estimation: ESPRIT”, IEEE Trans. Commun., vol. 48, no. 9, pp. 14591461, Sep. 2000.

[6] X. Ma, C. Tepedelenlioglu, G. B. Giannakis, and S. Barbarossa, "Nondata-aided carrier offset estimators for OFDM with null subcarriers identifiability, algorithms, and performance", IEEE J. Sel. Areas Commun., vol. 19, no. 12, pp. 2504-2515, Dec. 2001.

[7] S. Barbarossa, M. Pompili, and G. Giannakis, "Time and frequency synchornization of orthogonal frequency division multiple access systems", IEEE Int. Conf. Commun., vol. 6, pp. 1674-1678, Jun. 2001.

[8] Z. Shang, X. Mao, and J. Zhu, "A novel frequency synchronization method for wireless OFDM systems", in Vehicular Technology Conference, vol. 7, pp. 4824-4827, Sep. 2000.

[9] P. H. Moose, "A technique for orthogonal frequency division multiplexing frequency offset correction", IEEE Trans. Commun., vol. 42, no. 10, pp. 2908-2914, Oct. 1994.

[10] M. Luise and R. Reggiannini, "Carrier frequency acquisition and tracking for OFDM systems", IEEE Trans. Commun., vol. 44, no. 11, pp. 1590-1598, Nov. 1996.

[11] T.M. Schmidl and D. C. Cox, "Robust frequency and timing synchronization for OFDM", IEEE Trans. Commun., vol. 45, no. 12, pp. 1613-1621, Dec. 1997.

[12] M. Morelli and U. Mengali, "An improved frequency offset estimator for OFDM applications", IEEE Commun. Lett., vol. 3, no. 3, pp. 75-77, Mar. 1999. 